\title{
Purification, inhibitory properties, amino acid sequence and identification of the reactive site of a new serine proteinase inhibitor from oil-rape (Brassica napus) seed
}

\author{
Fabrizio Ceciliani ${ }^{\mathrm{a}, \mathrm{e}}$, Fabrizio Bortolotti ${ }^{\mathrm{b}}$, Enea Menegatti ${ }^{\mathrm{b}}$, Severino Ronchi ${ }^{\mathrm{a}, \mathrm{e}, *}$, Paolo Ascenzi ${ }^{\mathrm{c}}$, \\ Sandro Palmieri ${ }^{\mathrm{d}}$ \\ ${ }^{a}$ Istituto di Fisiologia Veterinaria e Biochimica, Università di Milano, via Celoria 10, 20133 Milan, Italy \\ ${ }^{b}$ Dipartimento di Scienze Farmaceutiche, Università di Ferrara, Via Fossato di Mortara 17/19, 44100 Ferrara, Italy \\ ${ }^{\circ}$ Dipartimento di Scienza e Tecnologia del Farmaco, Università di Torino, via Pietro Giuria 9, 10125 Turin, Italy \\ 'Istituto Sperimentale per le Colture Industriali, MAF, Via di Corticella 133, 40129 Bologna, Italy \\ ${ }^{\circ}$ Centro Interuniversitario per lo Studio delle Macromolecole Informazionali (CISMI), Milan, Italy
}

Received 25 January 1994; revised version received 1 March 1994

\begin{abstract}
A new serine proteinase inhibitor, rapeseed trypsin inhibitor (RTI), has been isolated from rapeseed (Brassica napus var. oleifera) seed. The protein inhibits the catalytic activity of bovine $\beta$-trypsin and bovine $\alpha$-chymotrypsin with apparent dissociation constants of $3.0 \times 10^{-10} \mathrm{M}$ and $4.1 \times 10^{-7} \mathrm{M}$, at $\mathrm{pH} 8.0$ and $21^{\circ} \mathrm{C}$, respectively. The stoichiometry of both proteinase-inhibitor complexes is 1:1. The amino acid sequence of RTI consists of 60 amino acid residues, corresponding to an $M_{\mathrm{r}}$ of about $6.7 \mathrm{kDa}$. The $\mathrm{P}_{1}-\mathrm{P}_{1}{ }^{\prime}$ reactive site bond has been tentatively identified at position Arg ${ }^{20}-\mathrm{Il} \mathrm{e}^{21}$. RTI shows no similarity to other serine proteinase inhibitors except the low molecular weight mustard trypsin inhibitor (MTI-2). RTI and MTI-2 could be members of a new class of plant serine proteinase inhibitors.
\end{abstract}

Key words: Serine proteinase inhibitor; Amino acid sequence; Rapeseed; Brassica napus var. oleifera

\section{Introduction}

Plants and seeds contain considerable amounts of proteins and peptides inhibiting serine proteinase activity. Their physiological roles include the regulation of endogenous proteinases during seed dormancy and, consequently, the mechanism of reserve protein mobilization [1]. Moreover, they are generally thought to contribute to the defence of plants against pathogens and herbivorous insects via inhibition of their proteolytic enzymes

\footnotetext{
* Corresponding author. Centro Interuniversitario per lo Studio delle Macromolecole Informazionali (CISMI), Milan, Italy. Fax: (39) (2) 236 7788 .

Abbreviations: RTI, rapeseed trypsin inhibitor III; MTI-2, low molecular weight mustard trypsin inhibitor; Bz-L-Arg-pNA, $N-\alpha$-benzoyl-Larginine-p-nitroanilide; Z-L-Tyr-ON $p, N$ - $\alpha$-carbobenzoxy-L-tyrosinepara-nitrophenyl ester; TFA, trifluoroacetic acid; trypsin, bovine $\beta$ trypsin; chymotrypsin, bovine $\alpha$-chymotrypsin; TPCK-trypsin, bovine trypsin treated with tosyl-L-phenylalanine chloromethyl ketone; TLCK-chymotrypsin, Bovine chymotrypsin trated with $N$ - $\alpha$-tosyl-Llysine chloromethyl ketone.
}

The protein sequence reported here has been submitted to the EMBL Data Bank with Accession No. P80301.
[2]. Due to their very high content of cysteine, serine proteinase inhibitors may also act as a storage or reserve protein [3]. Serine proteinase inhibitors have been investigated primarly in Graminaceae, Leguminosae and Solanaceae and, in general, are grouped in the soybean Kunitz, the Bowmann-Birk and the potato inhibitor families.

Different types of serine proteinase inhibitors have been identified in the same plant, suggesting that these proteins have evolved separately to perform distinct physiological roles. The seed of oil seed rape (Brassica napus var. oleifera) provides an interesting model for investigating these different functions since, at present, at least three distinct inhibitors have been identified from this source. Indeed, Inhibitor I, Inhibitor II, and Inhibitor III represent, respectively, 4-10\%, $13-33 \%$ and $60-$ $80 \%$ of the total inhibitory activity [4]. Inhibitors I and II are thermolabile, and have an apparent $M_{\mathrm{r}}$ higher than that of Inhibitor III, which is thermostable. The present study describes the purification, primary structure, and inhibitory properties of Inhibitor III from Brassica napus var. oleifera (RTI) and the tentative identification of the reactive site. Since the primary structure of this inhibitor excludes it from any of the families within the current classification of inhibitors, we propose that RTI and a 
similar low molecular weight trypsin inhibitor from white mustard seed (MTI-2) [5] belongs to a family of serine proteinase inhibitors not described yet.

\section{Experimental}

\subsection{Materials}

Seeds of a commercial variety (cv Anima) of oil seed rape (Brassica napus var. oleifera) were purchased from Semundo AG.

TPCK-trypsin was prepared from commercially available enzyme preparations (Sigma Chemical Co) according to Luthy et al. [6]. TLCKchymotrypsin, Bz-L-Arg-pNA, Z-L-Tyr-ONp, Tris, $p$-chloromercuribenzoate and 4-vinylpyridine were from Sigma Chemical Co. TFA and $6 \mathrm{~N} \mathrm{HCl}$ were from Pierce Chemical Co. Endoproteinase Lys-C was from Boehringer Mannheim. All chemicals used were of the highest purity commercially available.

\subsection{Purification procedure of $R T T$}

Ripe seeds of oil seed rape were homogenized in distilled water and centrifuged. The supernatant was then heated at $80^{\circ} \mathrm{C}$ for $3 \mathrm{~min}$. After cooling, the denaturated proteins were removed by centrifugation and the supernatant was lyophilized. The lyophilized crude inhibitor preparation was then dissolved in $120 \mathrm{ml}$ of $0.05 \mathrm{M}$ triethylamine formate buffer, pH 3.8, and loaded onto an SP-Sephadex G-25 column $(2.5 \times 28$ $\mathrm{cm}$ ) equilibrated with the same buffer. The column was washed with $500 \mathrm{ml}$ of the same buffer and the inhibitor was eluted with a linear gradient of 0.35 to $1 \mathrm{M} \mathrm{NaCl}$ in the starting buffer.

The active fractions were concentrated and loaded onto a Sephadex $\mathrm{G}-50 \mathrm{~F}$ column equilibrated with $0.01 \mathrm{M} \mathrm{KP}_{\mathrm{i}}, \mathrm{pH} 7.2$. Active fractions were pooled, dalyzed against $0.01 \mathrm{M} \mathrm{KP}, \mathrm{pH} 7.2$ and finally chromatographed on a CM-Sephadex G-25 column $(2.5 \times 14 \mathrm{~cm})$ equilibrated with $0.01 \mathrm{M} \mathrm{KP}_{\mathrm{i}}, \mathrm{pH} 7.2$. The inhibitor was eluted with $0.2 \mathrm{M} \mathrm{KCl}$ in the starting buffer. Active fractions were lyophilized and finally desalted on a Sephadex G-10 column equilibrated with $1 \%$ formic acid. RTI was further purified by RP-HPLC on a Vydac C-18 column $(0.46 \times 25 \mathrm{~cm})$, using a linear gradient from 20 to $40 \%$ acetonitrile in $0.16 \%$ heptafluorobutyric acid-triethylamine $(\mathrm{pH} \mathrm{3.0)}$ over $60 \mathrm{~min}$ at a flow rate of $1 \mathrm{ml} / \mathrm{min}$.

\subsection{Localisation of the reactive site}

To identify the inhibitory reaclive site, the lyophilized crude inhibitor preparation was treated with immobilized trypsin by loading onto a trypsin-Sepharose 4B column as described in [5]. Adsorbed material was eluted with $0.3 \mathrm{M} \mathrm{KCl}(\mathrm{pH} 1.8)$. Active fractions were desalted and further purified by RP-HPLC as reported above.

\subsection{Miscellaneous methods}

The apparent dissociation equilibrium constants $\left(K_{d}\right)$ for the binding of RTI to trypsin and chymotrypsin were determined by measuring its inibition on the protease activity using Bz-L-Arg-pNA and Z-L-Tyr$\mathrm{ON} p$ as substrates respectively [5], at $\mathrm{pH} 8.0$ and $21^{\circ} \mathrm{C}$.

Most of the methodologies used to determine the primary structure of RTI , that is S-pyridylethylation, digestion with endoproteinase Lys-C, RP-HPLC purification of the peptides and N-terminal amino acid sequencing were performed as described in [5].

Amino acid analysis was performed after gas-phase hydrolysis [5] by pre-column derivatization with 6-aminoquinolyl- $N$-hydroxysuccinimidyl carbamate [7] using a JASCO HPLC equipped with a 820 -FP detector.

Similarities between the primary structure of RTI and other proteins were searched using the Swiss Prot Protein Data Bank.

Capillary electrophoresis analysis on RTI was performed using an Model 270 HT capillary electrophoresis apparatus (Applied Biosystems) fitted with a $72 \mathrm{~cm} \times 50 \mathrm{~mm}$ I.D. capillary ( $50 \mathrm{~cm}$ to the detector). Free zone electrophoresis of RTI $(0.05 \mathrm{mg} / \mathrm{ml})$ was performed after precoating the capillary with Microcoat (Applied Biosystems) at $20 \mathrm{kV}$ $(20 \mathrm{~mA})$ for $15 \mathrm{~min}$ using $40 \mathrm{mM}$ sodium acetate $(\mathrm{pH} 4.0)$ as electrolyte.

\section{Results}

\subsection{Purification and determination of the primary structure of $R T I$}

RTI retains both anti-tryptic and anti-chymotryptic activities after heating at $80^{\circ} \mathrm{C}$ for $3 \mathrm{~min}$. Thus, taking advantage of the thermal stability of this inhibitor with respect to other trypsin inhibitors present in rapeseed

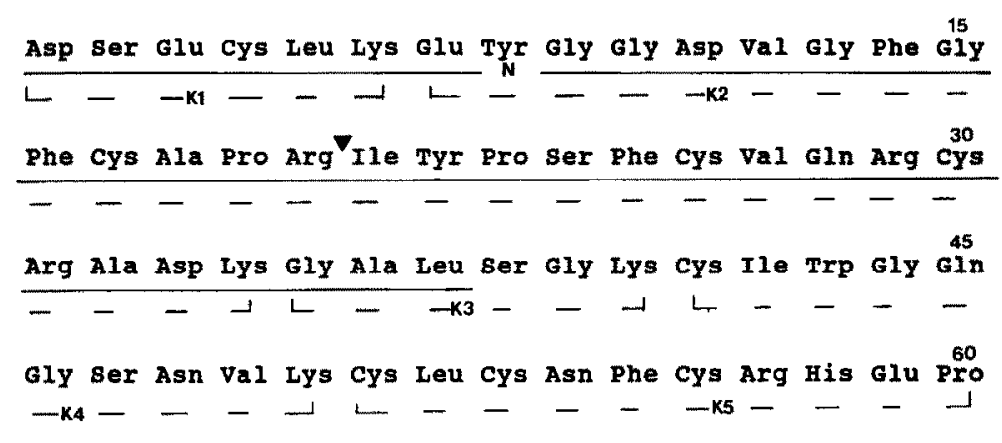

A

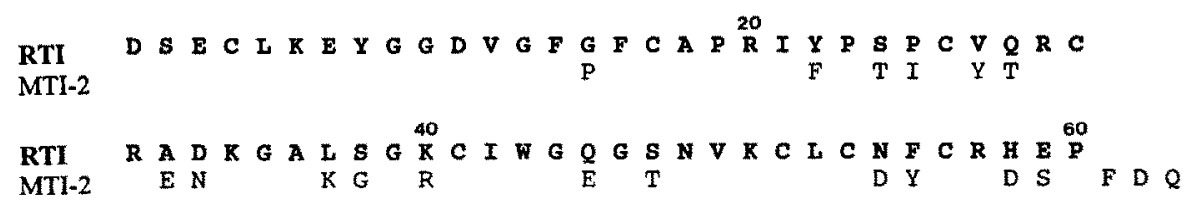

Fig. 1. The primary structure of RTI. (A) The continuous line indicates the sequence derived from amino-terminal sequencing of the entire protein. The dashed lines indicate the sequence derived from endoproteinase Lys-C digestion. The arrow indicates the $\mathrm{Arg}^{20}-\mathrm{Ile}^{21}$ bond cleaved by immobilized trypsin used as ligand for the affinity chromatography purification of the inhibitor (see text for details). (B) Sequence alignment between the primary structure of RTI and MTI-2. Only the replacements are indicated. 


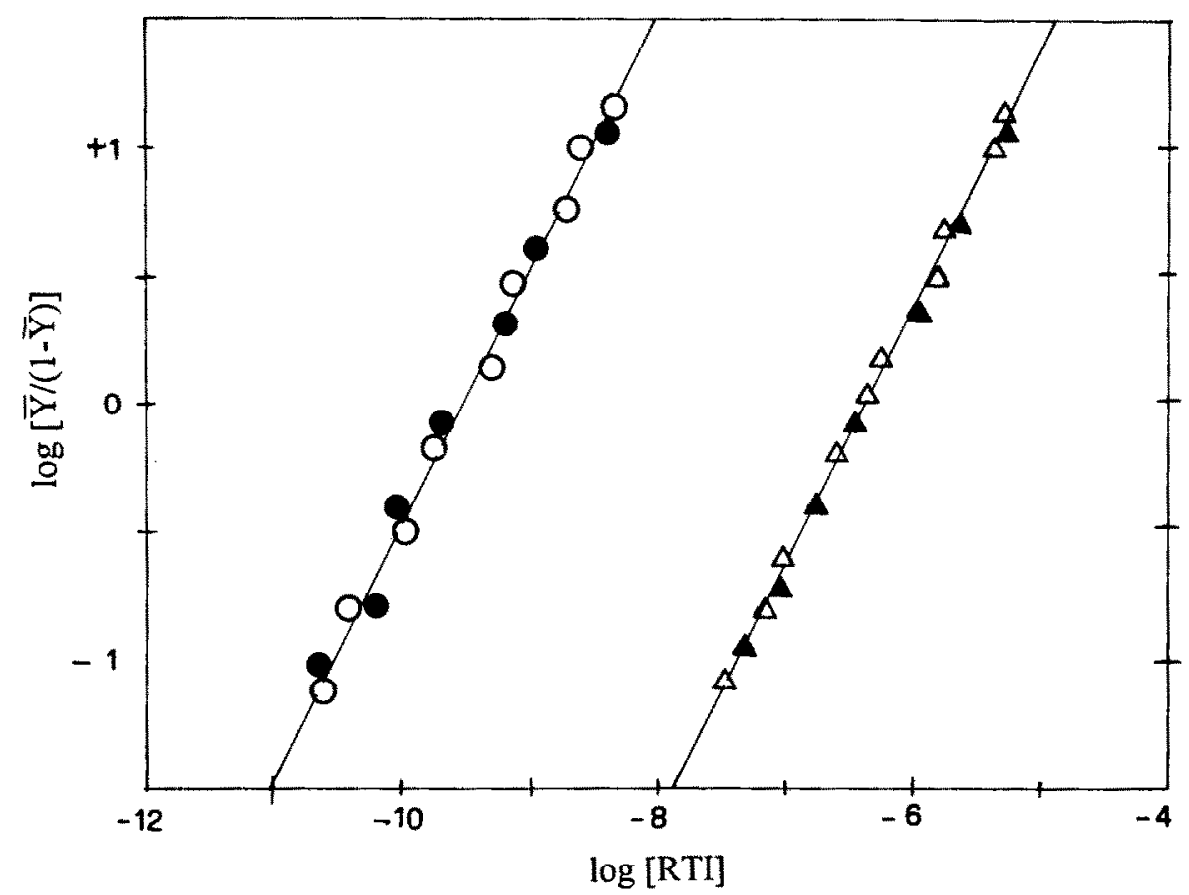

Fig. 2. Amount of RTI bound to trypsin $(O ; \bullet)$ and chymotrypsin $(\triangle ; \boldsymbol{\Delta})$ as a function of the free inhibitor concentration (expressed in M). Open and filled symbols refer to RTI and RTI after treatment with Immobilised trypsin, respectively. Solid lines were generated by the equation [6]:

$\overline{\mathrm{Y}}=1 /\left[1+\left(K_{\mathrm{d}} /[\mathrm{RTI}]\right)\right]$

using $K_{\mathrm{d}}=(3.0 \pm 0.2) \times 10^{-10} \mathrm{M}$ and $K_{\mathrm{d}}=(4.1 \pm 0.3) \times 10^{-7} \mathrm{M}$ for trypsin:RTI and chymotrypsin:RTI complex dissociation, respectively. For both serine-proteinase:inhibitor systems, the value of the Hill coefficient $(n)$ was $1.00 \pm 0.02$. values of $K_{\mathrm{d}}$ were obtained by iterative non-linear least-squares curve fitting. Data were obtained in $0.1 \mathrm{M}$ Tris- $\mathrm{HCl}, \mathrm{pH} 8.0$ and $21^{\circ} \mathrm{C}$.

seed, incorporation of heating step into the purification procedure facilitated the isolation of RTI.

Fig. 1A summarizes the data used to determine the complete primary structure of RTI. The protein consists of 60 amino acid residues, and the calculated 6592 molecular mass is in good agreement with the values determined by SDS-PAGE and gel-filtration $(6.5 \pm 0.5 \mathrm{kDa})$. The amino acid composition deduced from the sequence agrees well with that determined experimentally. Occasionally, some batches yielded RTIs lacking the N-terminal Asp. The absence of free thiol groups in RTI, determined by the $p$-chloromercuribenzoate procedure [8], supports the presence of four disulphide bridges in the molecule.

\subsection{Inhibitory properties of RTI and identification of the reactive site of $R T I$}

Fig. 2 shows the isotherms for RTI binding to trypsin and chymotrypsin using Bz-L-Arg- $p \mathrm{NA}$ and Z-L-Tyr$\mathrm{ON} p$ as substrates, respectively, at $\mathrm{pH} 8.0$ and $21^{\circ} \mathrm{C}$. Values of the apparent dissociation constant $\left(K_{\mathrm{d}}\right)$ for the formation of trypsin:RTI and chymotrypsin:RTI complexes were found to be $3.0 \times 10^{-10} \mathrm{M}$, and $4.1 \times 10^{-7} \mathrm{M}$, respectively. Values of $K_{\mathrm{d}}$ for the binding of native and trypsin-treated RTI to trypsin and chymotrypsin were indistinguishable. Moreover, the apparent stoichiometry for the complex formation was $1: 1$ in both cases. As expected for simple systems, values of $K_{d}$ were independent of enzyme and substrate concentrations, the apparent Hill coefficient being $1.00 \pm 0.02$.

The reactive site was identified after treatment with immobilized bovine trypsin, since the immobilized protease used as chromatographic ligand can hydrolyze the inhibitors at the reactive site $[9,10]$. Capilliary electropherogram of the native inhibitor trated with immobilized trypsin shows a single peak, whereas after reduction and $S$-pyridylethylation the same protein elutes as two peaks, with a different $\mathrm{Rf}$. These results clearly indicate that the immobilized trypsin cleaves the inhibitor into two polypeptides chains, which are linked by disulfide bridges in native protein. As expected, the amino-terminal sequence of RTI after immobilized trypsin treatment resulted in two amino acids released for each degradation cycle, in agreement with a first sequence starting from $A s p^{1}$ and ending at $\mathrm{Arg}^{20}$ and a second scquence starting from $\mathrm{Ile}^{21}$ and ending at Pro ${ }^{60}$. All these results confirm that the immobilized trypsin treatment causes a cleavage of the peptide bond at position $\mathrm{Arg}^{20}-\mathrm{Ile}^{21}$.

\section{Discussion}

The present study describes the purification, biochemical properties and structural characterization of a serine 
proteinase inhibitor from rapeseed seed, RTI, which is the first serine-proteinase inhibitor to be isolated and characterized from Brassica napus var. oleifera. The complete amino acid sequence of RTI has been determined and consists of 60 residues. Comparison of the primary structure of RTI with those in the Swiss Prot Protein Data Bank revealed that the sequence of RTI differs from that of other trypsin inhibitors, with the exception of MTI-2, an inhibitor from white mustard seed, which is not assignable to any of the families in the current inhibitor classification [5].

A total of 43 residues out of $60(70 \%)$ are identical to MTI-2 (Fig. 1B). Since all cysteine residues in RTI and MTI-2 are conserved and involved in disulfide bridges, we can suggest that the three-dimensional structures of the two inhibitors is consistent with a common protein fold. In this respect, the positions of the cysteine residues in RTI and MTI-2 are partly reminescent of those found in erabutoxin, although more structural data is required to confirm whether an agglutinin/neurotoxin-like fold is compatible with these two serine proteinase inhibitors.

The inhibitory activities of RTI and MTI-2 are very similar: values of $K_{\mathrm{d}}$ for the formation of serine proteinase:RTI complex $\left(3.0 \times 10^{-10} \mathrm{M}\right.$, and $4.1 \times 10^{-7} \mathrm{M}$ for trypsin and chymotrypsin, respectively) are in excellent agreement with those observed for MTI-2 binding to trypsin $\left(K_{\mathrm{d}} 1.6 \times 10^{-10} \mathrm{M}\right)$ and chymotrypsin $\left(K_{\mathrm{d}}\right.$ $5.0 \times 10^{-7} \mathrm{M}$ ) at $\mathrm{pH} 8.0$ and $21^{\circ} \mathrm{C}$.

As reported in section 3, the hydrolysis of the peptide bond $\mathrm{Arg}^{20}-\mathrm{Ile}^{21}$ of RTI does not affect inhibitory activity towards of trypsin and chymotrypsin action (Fig. 2). These findings are in accordance with the hypothesis that native and modified (i.e. with the $P_{1}-P_{1}{ }^{\prime}$ reactive site bond cleaved) serine proteinase inhibitors (e.g. Kunitz-type inhibitors) associate to trypsin and chymotrypsin with the same affinity [11], mantaining the enzyme inhibition (re)active site geometry virtually unperturbed [3].

The 17-26 polypeptide loop of RTI shows some particular structural properties, which are found in serine proteinase inhibitors reactive sites [3]. This region is strongly connected to the protein core by two disulphide bridges, i.e. $\mathrm{Cys}^{16}-\mathrm{Cys}^{35}$ and $\mathrm{Cys}^{24}-\mathrm{Cys}^{56}$ in Kazal type inhibitors: a similar role can be envisaged for $\mathrm{Cys}^{17}$ and $\mathrm{Cys}^{26}$ in RTI. Moreover, Pro $^{19}$, expected as the $\mathbf{P}_{2}$ residue of the RTI potential reactive site, finds a counterpart in Kunitz- and Kazal-type inhibitors, where $\operatorname{Pro}^{13}\left(\mathrm{P}_{3}\right)$ and Pro $^{17}\left(\mathrm{P}_{2}\right)$ respectively play a central role in the achievement of proper composition for the $\mathrm{P}_{1}-\mathrm{P}_{1}^{\prime}$ scissile peptide bond. An additional prolyl residue is present both in RTI and in Kazal-type inhibitor on the $\mathrm{P}^{\prime}$ side of the reactive peptide ( $\operatorname{Pro}^{23}\left(\mathrm{P}_{3}{ }^{\prime}\right)$ and $\operatorname{Pro}^{22}\left(\mathrm{P}_{4}{ }^{\prime}\right)$, respectively).

Thus, these considerations confirm the experimental data reported in this paper which suggest that $\operatorname{Arg}^{20}$ and $1 \mathrm{e}^{21}$ side chains may be envisaged as the $P_{1}$ and $P_{1}{ }^{\prime}$ inhibitor reactive site residues in RTI interacting with the $S_{1}$ and $S_{1}{ }^{\prime}$ subsites of trypsin and chymotypsin.

The presence of several serine-proteinase inhibitors in the same plant might be justified by the need of the plant to have a defensive pattern capable of being modulated against distinct proteolytic enzymes of different pathogen species. Norioka et al. [13] proposed a second hypothesis, suggesting that the inhibitors belonging to the Kunitz family have been gradually replaced within the course of evolution by a more thermostable one, which in Leguminosae has been identified as Bowman-Birk family inhibitor. Nevertheless, in Brassicaeae, a new thermostable proteinases inhibitor family, represented by RTI and MTI-2, may have been selected. The preponderance of thermostable serine-proteinase inhibitors should be considered a desirable trait that improves the genetic resistance of plants toward parasites.

Acknowledgments: The authors thank Prof. Armando Negri and Prof. Martino Bolognesi for helpful discussions. This study was supported by financial contributions from the Italian Ministry of University, Scientific Research and Technology (MURST), of the Italian Ministry of Agriculture, Food and Forestry Resources (MRAFF), target oriented project ' $R$ esistenze genetiche delle piante agrarie agli stess biotici ed abiotici, sotto-progetto No. 17' and the Italian Research Council (CNR), target oriented project 'Chimica Fine II'.

\section{References}

[1] Richardson, M. (1981) Food. Chem. 6, 235-253.

[2] Broadway, R. (1993) Phytochemistry 33, 21-27.

[3] Bode, W. and Huber, R. (1991) Eur. J. Biochem. 204, 433-451.

[4] Visintin, M., Iori, R., Valdicelli, L. and Palmieri, S. (1992) Phytochemistry $31,3677-3680$

[5] Menegatti, E., Tedeschi, G., Ronchi, S., Bortolotti, F., Ascenzi, P., Thomas, R.M., Bolognesi, M. and Palmieri, S. (1992) FEBS Lett. 301, 10-14.

[6] Luthy, J.A., Praissman, M., Finkestadt, W.R. and Laskowski Jr., M. (1973) J. Biol. Chem. 248, 1760-1771

[7] Cohen, S. and Michaud, D.P. (1993) Anal. Biochem. 211, 279-287.

[8] Boyer, P.D. (1954) J. Am. Chem. Soc. 76, 4331-4337.

[9] Wilimowska-Pelc, A. (1989) Acta Societatis Botanicorim Poloniae $58,563-573$.

[10] Ryan, C.A. (1981) in: The Biochemistry of Plants, Vol. 9, pp. 351-370, Academic Press, New York.

[11] Tschesche, H. and Kupfer, D. (1976) Hoppe-Seyler's Z. Physiol. Chem. 357, 769-776.

[12] Norioka, N., Hara, R., Ikenaka, T. and Abe, J. (1988) Agric. Biol. Chem. 52, 1245. 